\title{
Correlates of transient versus persistent psychotic symptoms among dependent
}

methamphetamine users

Running title: Methamphetamine use and psychosis

*Rebecca McKetin ${ }^{\mathrm{a}, \mathrm{b}}$, Jonathon Gardener ${ }^{\mathrm{a}}$, Amanda L. Baker ${ }^{\mathrm{c}}$, Sharon Dawe ${ }^{\mathrm{d}}$, Robert Alie , Alexandra Voce ${ }^{\mathrm{a}}$ and Liana Leach ${ }^{\mathrm{a}}$ and Dan I. Lubman ${ }^{\mathrm{f}}$

${ }^{\mathrm{a}}$ Centre for Research on Ageing, Health and Well-being, the Australian National University, Canberra, Australia

${ }^{\mathrm{b}}$ National Drug and Alcohol Research Centre, University of New South Wales, Sydney,

Australia

${ }^{\mathrm{c}}$ Priority Research Centre for Translational Neuroscience and Mental Health, University of Newcastle, Callaghan, Australia

${ }^{d}$ School of Applied Psychology, Menzies Health Institute Queensland, Griffith University, Brisbane, Australia

${ }^{\mathrm{e}}$ University of Adelaide, Adelaide, Australia

${ }^{6 f}$ Turning Point, Eastern Health and Monash University, Melbourne, Australia

Word count: 3,566 (excluding abstract and references), 20 pages, 1 Table

*Corresponding author:

Rebecca McKetin, Centre for Research on Ageing, Health and Well-being, Building 54 Mills Road (Florey), The Australian National University, Acton 2601 ACT, Australia Ph. + 61261258407

Fax. +61261251558

Email: rebecca.mcketin@anu.edu.au 


\begin{abstract}
This study examined correlates of transient versus persistent psychotic symptoms among people dependent on methamphetamine. A longitudinal prospective cohort study of dependent methamphetamine users who did not meet DSM-IV criteria for lifetime schizophrenia or mania. Four non-contiguous one-month observation periods were used to identify participants who had a) no psychotic symptoms, $(\mathrm{n}=110)$; (b) psychotic symptoms only when using methamphetamine (transient psychotic symptoms, $\mathrm{n}=85$ ); and, (c) psychotic symptoms both when using methamphetamine and when abstaining from methamphetamine (persistent psychotic symptoms, $\mathrm{n}=37$ ). Psychotic symptoms were defined as a score of 4 or greater on any of the Brief Psychiatric Rating Scale items of suspiciousness, hallucinations or unusual thought content. Relative to no psychotic symptoms, both transient and persistent psychotic symptoms were associated with childhood conduct disorder and comorbid anxiety disorders,. Earlier onset methamphetamine use and being male were more specifically related to transient psychotic symptoms, while a family history of a primary psychotic disorder and comorbid major depression were specifically related to persistent psychotic symptoms. We conclude that there are overlapping but also distinct clinical correlates of transient versus persistent psychotic symptoms, suggesting potentially heterogeneous etiological pathways underpinning the psychotic phenomena seen amongst people who use methamphetamine.
\end{abstract}

Keywords: street drugs, central nervous system stimulants, amphetamine, amphetaminerelated disorders, psychotic disorders, paranoia, hallucinations 


\section{Introduction}

Methamphetamine ('crystal meth' or 'ice') is an addictive synthetic stimulant that can produce a brief psychotic episode, characterized by persecutory delusions and hallucinations, which strongly resembles acute paranoid schizophrenia (Angrist \& Gershon, 1970; Bell, 1973; Griffith, 1970). The risk of psychosis is dose-related, increasing during periods of heavy methamphetamine use and receding during periods of abstinence (McKetin et al., 2013) and can be exacerbated by premorbid concurrent risk factors for psychosis (e.g. comorbid disorder, stress, sleep deprivation)(Bramness et al., 2012; Chen et al., 2003; Glasner-Edwards $\&$ Mooney, 2014). However, not all people who use methamphetamine experience psychotic symptoms (Chen et al., 2003; Hides et al., 2015). Among those who do, most experience a transient psychosis that recedes after intoxication from methamphetamine (Chen et al., 2003; Schuckit, 2006). A minority of people experience a more prolonged psychosis that persists beyond the use of the drug (Akiyama, 2006; Iwanami et al., 1994) and vulnerability to recurrent psychotic episodes that can occur in the absence of methamphetamine use (Sato, 1983). It is unclear whether this persistent psychosis reflects the precipitation of a schizophrenia spectrum disorder in vulnerable individuals (Callaghan et al., 2012; NiemiPynttari et al., 2013; Schuckit, 2006) or a chronic form of methamphetamine-induced psychosis (Tomiyama, 1990).

Identifying risk factors for methamphetamine-related psychosis, particularly the persistent form, is a necessary first step in providing targeted health promotion messages to people who use the drug and early interventions for those who may be at risk of developing a chronic psychosis. In addition to heavier methamphetamine use (Chen et al., 2003; Degenhardt et al., 2015; Hall et al., 1996; Lecomte et al., 2013; McKetin et al., 2013; McKetin et al., 2006), identified correlates of methamphetamine-related psychosis include initial use at a young age, a premorbid schizoid personality, a family history of schizophrenia (Chen et al., 2005; Chen et al., 2003; Tsuang et al., 1982) or affective illness (Chen et al., 2005), childhood adversity 
(Ding et al., 2014) and behavioral problems (Salo et al., 2013), and comorbid mental disorders, including major depression, alcohol dependence and antisocial personality disorder (Chen et al., 2003; Lecomte et al., 2013).

Less is known about factors that predict the more persistent form of methamphetamine psychosis and inconsistent findings have been reported from the few studies that have systematically examined correlates of transient versus persistent methamphetamine psychosis. On the one hand, persistent psychosis has been associated with greater premorbid risk for schizophrenia (Chen et al., 2005; Chen et al., 2003), depression, antisocial personality traits, and a longer history of substance use (Lecomte et al., 2013). On the other hand, other studies have not found a link between methamphetamine-related psychosis and a premorbid risk for schizophrenia (Hides et al., 2015; Lecomte et al., 2013; McKetin et al., 2006), nor have they consistently reported on whether risk factors (e.g. depression, substance use history) differ for transient versus persistent psychosis (Chen et al., 2005; Chen et al., 2003).

In our past research we utilized a longitudinal study design to understand how methamphetamine use increases risk for experiencing psychotic symptoms. Specifically, by tracking a cohort of dependent methamphetamine users (who did not meet lifetime DSM-IV criteria for schizophrenia or mania), we were able to document a dose-related increase in the occurrence of psychotic symptoms during periods of methamphetamine use relative to periods of abstinence (McKetin et al., 2013). Although most symptoms of psychosis in this cohort were transient, a minority of participants reported psychotic symptoms during periods when they were not using the drug, consistent with the notion of a more persistent form of methamphetamine psychosis. It was also apparent that, although methamphetamine substantially increased the risk of psychotic symptoms, not all people who used the drug experienced psychotic symptoms. 
In this paper, we explore potential clinical and demographic correlates associated with the expression of psychotic symptoms in this cohort of dependent methamphetamine users. Specifically, we examined whether there were any differences between people who experienced psychosis exclusively when using methamphetamine, those who continued to experience psychosis when they stopped using the drug, and those who did not experience psychotic symptoms at all.

\section{Method}

\subsection{Participants and procedure}

Participants ( $\mathrm{N}=278)$ all met DSM-IV criteria for methamphetamine dependence on entry to the study and none met DSM-IV criteria for lifetime schizophrenia or mania. DSM-IV diagnoses were assessed using the Composite International Diagnostic Interview (CIDI) (Andrews \& Peters, 1998). Participants were from a larger study, the Methamphetamine Treatment Evaluation Study (MATES) cohort, which is detailed elsewhere (McKetin et al., 2012). In brief, the MATES cohort included 400 people entering community-based drug treatment services in Sydney and Brisbane, Australia, for methamphetamine use, and 101 methamphetamine users from Sydney who were not in treatment (i.e., recruited through needle and syringe programs and outreach services) but who screened positive for dependence on methamphetamine. Other inclusion criteria for MATES were being at least 16 years old, comprehension of English, being willing to participate in follow-up interviews, and, not having been in methamphetamine treatment, other inpatient drug treatment, or in prison, in the month prior to entering the study. These latter exclusion criteria were necessary in MATES to obtain a naturalistic baseline measure of drug use.

From the MATES cohort, 17 participants were excluded from the current study because they did not meet DSM-IV criteria for methamphetamine dependence on recruitment. A further 59 
were excluded because they met DSM-IV criteria for either lifetime schizophrenia or a lifetime manic episode, and 138 were excluded because this diagnostic information was not available (i.e., these participants did not partake in the follow-up interviews when diagnoses were made). A further 9 participants were excluded because they had not used methamphetamine during any of the one-month observation periods used in the analysis.

A structured interview schedule was administered at baseline and each follow-up ( 3 months, 1 year and 3 years after the baseline interview). Recruitment of the cohort took place in 2006 and 2007, while follow-up interviews spanned the period from 2006 to 2010. Interviews were conducted face-to-face or by phone. All participants provided informed consent, were volunteers, and were reimbursed for their time and travel expenses (up to AUD40 per interview). All of the participants in the current study were re-interviewed at 3 months and 1 year after entry to the cohort, and $83 \%(\mathrm{n}=230)$ were interviewed at 3 years. The current study used data on drug use and psychotic symptoms in the past month at each of these four time points, totaling 1,064 months of data for all of the participants combined.

The study was approved by the UNSW Human Research Ethics Committee and this permission was ratified by all other participating institutions. All participants were volunteers who provided informed consent prior to participation and who were reimbursed up to AU $\$ 40$ per interview.

\subsection{Measures}

2.2.1 Psychotic symptoms: Psychotic symptoms were assessed for the past month and were defined as a score of four or greater on any of the Brief Psychiatric Rating Scale (BPRS) items of suspiciousness, unusual thought content or hallucinations. BPRS scores of 4+ indicate clinically significant or pathological symptom intensity (Lukoff et al., 1986). Ratings 
were made by trained interviewers (Honours level psychology graduates or equivalent) and weekly meetings were held to review BPRS ratings in order to maintain inter-rater agreement and avoid rater drift (Ventura et al., 1993). Inter-rater agreement for the definition of psychotic symptoms used in this study was 93\%, yielding a kappa of 0.86 (McKetin et al., 2013).

2.2.2 Methamphetamine use: Days of methamphetamine use in the past four weeks was measured using the Opiate Treatment Index (Darke et al., 1992). Self-reported abstinence from methamphetamine use was confirmed in a sub-sample of the entire MATES cohort, with false reporting of abstinence occurring in only $6 \%$ of cases (McKetin et al., 2013). Age of first methamphetamine use, route of methamphetamine administration, and severity of methamphetamine dependence were assessed at baseline. Severity of methamphetamine dependence was assessed using the Severity of Dependence Scale (SDS), a validated five item scale that yields a score between 0 and 15, with higher scores reflecting more severe dependence (Gossop et al., 1995).

2.2.3 Other drug use measures: Days of use in the past four weeks was measured for cannabis, heroin, cocaine, ecstasy, hallucinogens, alcohol, tobacco, benzodiazepines and antidepressant medication. Both prescription and non-prescription use of benzodiazepines and antidepressant medication were included.

\subsubsection{Comorbidity: The CIDI (Andrews \& Peters, 1998) was used to make DSM-IV} diagnoses of methamphetamine dependence and other Axis I psychiatric disorders. Past year diagnoses of social phobia, panic disorder and major depression were made at the baseline and at the 1 year follow-up interview. A positive diagnosis was based on meeting diagnostic criteria at either of these two interviews. A DSM-IV diagnosis of childhood conduct disorder was made using a modified version of the Diagnostic Interview Schedule (Robins et al., 
1982). A history of Attention Deficit Hyperactivity Disorder (ADHD) was based on the participant reporting that they had ever been told by a doctor that they had this condition.

2.2.5 Family history of psychotic disorders: A family history of primary psychotic disorder was assessed by asking the participant "Has anyone in your family ever been diagnosed with a major mental illness, like mania, bipolar disorder, schizophrenia, or other psychosis?" Disorders were coded as schizophrenia, mania/bipolar, drug-induced psychosis, and other psychosis (e.g., depression with psychotic features, post-partum psychosis). Drug-induced psychotic disorders were not included as a primary psychotic disorder.

\subsection{Design and statistical analysis}

Data were analyzed using Stata SE version 11.2 (Stata Corporation, 2014). All tests were twosided with significance set at $\mathrm{p}<0.05$. An initial overall test was conducted to establish whether between-group differences existed across the three groups. Follow-up pairwise comparisons were conducted to establish which groups differed. These comparisons were made using a Kruskal Wallis test for continuous data (because these data were not normally distributed) and a Pearson's Chi-square test for categorical outcomes. Multivariate simultaneous logistic regression was used to adjust for between group differences in severity of methamphetamine dependence (SDS score) when comparing the persistent psychotic symptom group and no psychotic symptom group on family history of psychosis and comorbid depression. 


\section{Results}

\subsection{Characteristics of the sample}

Participants $(\mathrm{N}=278)$ had a mean age of 31.7 years $(\mathrm{SD}=8.1$ years $), 72 \%$ were male, $89 \%$ were Australian born and 96\% nominated English as their preferred language. All met DSMIV criteria for methamphetamine dependence on entry to the study; they had used the drug for a mean of 13.1 years ( $\mathrm{SD}=7.9$ years) and $83 \%$ had injected it. Methamphetamine use occurred during $58 \%$ of the observed months (on a median of 8 days, range 1-28 days) with injection the main route of administration (79\% of months). Other drug use consisted primarily of tobacco, alcohol and cannabis ( $89 \%, 62 \%$ and $57 \%$ of months respectively). Psychotic symptoms occurred for $25 \%$ of all months and $60 \%$ of participants reported psychotic symptoms during at least 1 month of their follow-up. Further details on the sample characteristics and the relationship between methamphetamine use and psychotic symptoms are reported elsewhere (McKetin et al., 2013).

\subsection{Transient and persistent psychotic symptoms in the sample}

Participants were divided into three groups according to whether they experienced psychotic symptoms: (a) neither during any months when they were using methamphetamine nor during any months when they were not using methamphetamine (no psychotic symptoms, $\mathrm{n}=110$, 47\%); (b) during at least one month when they were using methamphetamine, but not during any months when they were not using the drug (transient psychotic symptoms, $\mathrm{n}=85,37 \%$ ); and (c) during at least one month when they were using methamphetamine and also during at least one month when they were not using the drug (persistent psychotic symptoms, $\mathrm{n}=37$, 16\%). Participants were excluded from these groupings if they had used methamphetamine on all of the follow-ups because we could not confirm the absence of psychotic symptoms during periods of abstinence from methamphetamine $(n=35)$ or if they had psychotic symptoms only during months of abstinence from methamphetamine $(\mathrm{n}=11)$. 


\subsection{Correlates of vulnerability to psychotic symptoms}

There were overlapping and distinct factors associated with transient versus persistent psychotic symptoms (Table 1). Compared to participants who experienced no psychotic symptoms, participants reporting transient psychotic symptoms were more likely to have a history of conduct disorder and have a comorbid anxiety disorder. Persistent psychotic symptoms were similarly associated with conduct disorder and anxiety disorders (being more strongly associated with panic disorder than were transient psychotic symptoms).. Persistent psychotic symptoms were also associated with major depression and a family history of a primary psychotic disorder.

Groups were reasonably comparable in their drug use history on entry to the study, with the exception of participants with persistent psychotic symptoms being more severely dependent on methamphetamine (Table 1). The relationship between persistent psychotic symptoms and a family history of a psychotic disorder (adjusted OR 2.7, 95\% CI 1.1- 6.4, p $=0.026$ ) and comorbid depression (adjusted OR 2.8, 95\% CI 1.2- 6.8, $\mathrm{p}=0.021$ ) remained significant after adjusting for severity of methamphetamine dependence.

To investigate whether the use of substances other than methamphetamine contributed to persistent psychotic symptoms we examined substance use for months when participants were abstaining from methamphetamine (i.e., at risk of persistent psychotic symptoms). Compared to participants with no psychotic symptoms, participants with persistent psychotic symptoms were more likely to have taken antidepressants $\left(27 \%\right.$ vs. $\left.44 \%, \chi^{2}=4.3, p=0.039\right)$ and benzodiazepines $\left(12 \%\right.$ vs. $\left.24 \%, \chi^{2}=4.9, p=0.026\right)$ during these months when they were abstaining from methamphetamine. There were no other significant group differences in other substance use during these months, or during months of methamphetamine use $(p>0.05$, analyses not shown). 
Table 1 about here. 


\section{Discussion}

We found different clinical correlates of transient versus persistent psychotic symptoms in this otherwise homogeneous sample of dependent methamphetamine users. Although some factors were associated with both transient and persistent psychotic symptoms (childhood conduct disorder, comorbid anxiety disorders) a family history of a primary psychotic disorder, comorbid depression, and more severe dependence on methamphetamine, were specifically associated with persistent psychotic symptoms. Both a family history of a primary psychotic disorder and comorbid depression remained significantly associated with persistent psychotic symptoms after adjusting for severity of methamphetamine dependence.

Our findings are suggestive of potentially distinct but overlapping etiological pathways involved in the development of transient versus persistent psychotic symptoms in people who use methamphetamine. The disjuncture between predictors of transient and persistent psychotic symptoms is consistent with the findings of Tsuang et al., who found that longlasting substance-related psychosis was associated with a broader clinical symptom profile and different clinical correlates (more premorbid personality disorders, greater familial risk of schizophrenia and affective disorders) than brief substance-related psychosis (Tsuang et al., 1982). Our findings are also consistent with the broader research on psychotic experiences in the general population, in that, although there is evidence of a continuum of psychosis and related risk factors, there is evidence that there are distinct etiological influences on different psychotic experiences (e.g. higher heritability for negative symptoms cf. hallucinations) (McGrath et al., 2015; van Os, 2014; Zavos et al., 2014).

The finding that persistent psychotic symptoms were more specifically related to a family history of a primary psychotic disorder suggests that these individuals may have a preexisting vulnerability to psychosis. Past studies have also found also found greater familial risk for schizophrenia among people who experience lasting vs. brief psychosis in the context 
of methamphetamine use (Chen et al., 2005) and other substance use (Tsuang et al., 1982).

Taken together, these findings suggest that persistent psychosis may reflect the precipitation of schizophrenia, or unmasking of schizophrenia in vulnerable individuals, which may be etiologically distinct from the transient psychotic phenomena induced by acute use of the drug (Schuckit, 2006).

Our finding that major depression was specifically associated with persistent psychotic symptoms is consistent with elevated familial risk for affective disorders among people with long-lasting versus brief substance-related psychosis (Tsuang et al., 1982) and lasting depressive symptoms seen amongst methamphetamine users who experience persistent psychosis (Lecomte et al., 2013). Depression may form an intrinsic symptom of persistent methamphetamine psychosis, making it similar to schizoaffective disorder, or an atypical schizophrenia (Tsuang et al., 1982), or it could also reflect the affective prodrome seen with first episode psychosis (Birchwood, 2003). Comorbid depression could also be a consequence of experiencing prolonged psychosis because of the psychological distress caused by ongoing psychotic symptoms and the impact that this can have on a person's day-to-day functioning (Birchwood, 2003).

We found a strong relationship between anxiety disorders and both transient and persistent psychosis, suggesting that anxiety may play a role in the development of psychotic symptoms in methamphetamine users. The elevated prevalence of anxiety disorders, and their interplay with psychosis, has been recognized in the context of primary psychotic disorders (Birchwood, 2003; Cassano et al., 1998), but has not been documented for methamphetamine psychosis. Comorbidity between psychosis and anxiety disorders is likely driven by a bidirectional effect of paranoia increasing social fear and perception of social criticism, while the propensity toward such sensitivities in people with anxiety disorders may fuel paranoia by augmenting perceptions of threat (Grezellschak et al., 2015). The development of psychosis 
could further entrench the social avoidance seen with these disorders because of the stigma attached to psychosis and/or fear of other people (Birchwood, 2003). In the context of methamphetamine use, symptoms of anxiety and panic are likely to be further exacerbated by the physiological effects of stimulant intoxication, those including tachycardia, chest pain and sympathetic arousal. Increased recognition of anxiety disorders in people who use methamphetamine, and the use of cognitive therapies that target misperceptions of social threat (Grezellschak et al., 2015), may be helpful to alleviate both anxiety and the severity of psychotic symptoms.

Other correlates of psychotic symptoms were generally consistent with past research. Antisocial personality disorder and its childhood precursor, conduct disorder, are recognized comorbidities in people with psychotic disorders (Mueser et al., 2006), and our study is one of a growing number to identify this link with methamphetamine psychosis (Chen et al., 2003; Lecomte et al., 2013; Sulaiman et al., 2014). Although male gender was not significantly associated with psychosis risk, this null finding may have been due to lack of statistical power.

A limitation of our approach was that we relied on several discrete data collection epochs to judge whether participants had transient versus persistent psychotic symptoms. It is possible that a proportion of participants experienced persistent psychotic symptoms that were not captured within these discrete observation periods. This constraint would have resulted in the misclassification of some participants as having transient psychotic symptoms even though they may have experienced persistent symptoms. Similarly, a proportion of participants may have experienced transient psychotic symptoms, but not at the times that we observed them (and hence would have been misclassified as having no psychotic symptoms). This misclassification would have reduced our ability to detect differences between groups. Therefore, null effects found in this study, particularly where non-significant trends were 
apparent, should be treated tentatively. The small number of participants classified as having persistent psychosis may have also contributed to null findings. Replication of these results in a larger cohort would be desirable to confirm null effects.

The relatively small number of participants classified as having a persistent psychotic symptoms prevented a more sophisticated modelling of what factors were most predictive of persistent versus transient psychosis. Importantly, several of the predictors we examined could be spuriously associated with psychosis by virtue of their relationship with comorbid conditions (e.g., conduct disorder is more prevalent in males and associated with increased prevalence of other mental disorders (Nock et al., 2006)). Therefore it is not possible to make conclusive statements about which factors best predict psychosis compared with those that are merely correlates of the condition.

Finally, we only assessed the core symptoms of methamphetamine psychosis (paranoid delusions and hallucinations), whereas the broader symptom profile of transient and persistent substance-related psychosis have been found to differ (Caton et al., 2006; Tsuang et al., 1982). It would be helpful in future research to see whether distinct predictors of transient versus persistent methamphetamine psychotic symptoms were due to their association with specific symptoms that differed between these conditions. For example, Tsuang et al. found that long-lasting substance-related psychosis was characterized by more negative symptoms (e.g. poor insight, flat affect), greater delusional content, and fewer hallucinations than brief substance-related psychosis (Tsuang et al., 1982). Negative symptoms and paranoia have higher heritability than hallucinations (Zavos et al., 2014), which might explain why we found that persistent psychotic symptoms were more strongly related to family history of a primary psychotic disorder. In other words, correlates of transient versus persistent psychotic symptoms may reflect the specific symptom profile of these conditions rather than symptom duration per se. 
In summary, our findings emphasize that there are overlapping but also distinct clinical correlates of transient versus persistent psychotic symptoms among methamphetamine users, suggesting that these entities may be, to some extent, etiologically distinct. This finding is consistent with the broader psychosis literature where there is increasing recognition that different psychotic experiences/phenotypes may have different etiologies and clinical correlates (McGrath et al., 2015). Despite this complexity, many of our findings replicate previously identified correlates of methamphetamine psychosis risk. Understanding which factors predict a more chronic course of psychosis in methamphetamine users is a critical first step in identifying people who may benefit from early intervention to prevent a progression to schizophrenia.

\section{Acknowledgements}

The research was funded by the National Health and Medical Research Council (Project Grant No. 350974) and the Australian Government Department of Health and Ageing. Data were collected through the Methamphetamine Treatment Evaluation Study (MATES), conducted by the National Drug and Alcohol Research Centre, University of New South Wales. The authors acknowledge the contribution of other project investigators: Richard Mattick, Joanne Ross, Jake Najman and Nicole Lee. Thanks also go to the research officers who assisted with data collection (Shelley Cogger, Erin Kelly, Kate Hetherington, Grace Ho, Cathie Sammut, Julia Rosenfeld, Sagari Sarkar, Rachel Sutherland and Miriam Wyzenbeek), participating treatment agencies, and the research participants.

Role of funding source: The funding bodies played no role in the design of the study; the collection, analysis or interpretation of data; the writing of the report; or, the decision to submit the article for publication. 
Conflicts of interest: Dan Lubman has provided consultancy advice to Lundbeck, and has received travel support and speaker honoraria from Astra Zeneca, Bristol Myers Squibb, Janssen and Lundbeck. Robert Ali has received travel support and speaker honoraria from Reckitt Benckiser pharmaceutical. Other authors have no conflicts of interest. 


\section{References}

Akiyama, K., 2006. Longitudinal clinical course following pharmacological treatment of methamphetamine psychosis which persists after long-term abstinence. Ann N Y Acad Sci. 1074, 125-134.

Andrews, G., Peters, L., 1998. The psychometric properties of the Composite International Diagnostic Interview. Soc Psychiatry Psychiatr Epidemiol. 33, 80-88.

Angrist, B. M., Gershon, S., 1970. The phenomenology of experimentally induced amphetamine psychosis--preliminary observations. Biol Psychiatry. 2, 95-107.

Bell, D. S., 1973. The experimental reproduction of amphetamine psychosis. Arch Gen Psychiatry. 29, 35-40.

Birchwood, M., 2003. Pathways to emotional dysfunction in first-episode psychosis. Br J Psychiatry. 182, 373-375.

Bramness, J. G., Gundersen, O. H., Guterstam, J., Rognli, E. B., Konstenius, M., Loberg, E. M., Medhus, S., Tanum, L., Franck, J., 2012. Amphetamine-induced psychosis--a separate diagnostic entity or primary psychosis triggered in the vulnerable? BMC Psychiatry. 12, 221.

Callaghan, R. C., Cunningham, J. K., Allebeck, P., Arenovich, T., Sajeev, G., Remington, G., Boileau, I., Kish, S. J., 2012. Methamphetamine Use and Schizophrenia: A Population-Based Cohort Study in California. Am J Psychiatry. 169, 389-396.

Cassano, G. B., Pini, S., Saettoni, M., Rucci, P., Dell'Osso, L., 1998. Occurrence and clinical correlates of psychiatric comorbidity in patients with psychotic disorders. J Clin Psychiatry. 59, 60-68.

Caton, C. L. M., Hasin, D. S., Shrout, P. E., Drake, R. E., Dominguez, B., Samet, S., Schanzer, B., 2006. Predictors of psychosis remission in psychotic disorders that cooccur with substance use. Schizophr Bull. 32, 618-625.

Chen, C. K., Lin, S. K., Sham, P. C., Ball, D., Loh el, W., Murray, R. M., 2005. Morbid risk for psychiatric disorder among the relatives of methamphetamine users with and without psychosis. Am J Med Genet B Neuropsychiatr Genet. 136B, 87-91.

Chen, C. K., Lin, S. K., Sham, P. C., Ball, D., Loh, E. W., Hsiao, C. C., Chiang, Y. L., Ree, S. C., Lee, C. H., Murray, R. M., 2003. Pre-morbid characteristics and co-morbidity of methamphetamine users with and without psychosis. Psychol Med. 33, 1407-1414.

Darke, S., Hall, W., Wodak, A., Heather, N., Ward, J., 1992. Development and validation of a multi-dimensional instrument for assessing outcome of treatment among opiate users: the Opiate Treatment Index. Br J Addict. 87, 733-742.

Degenhardt, L., Coffey, C., Hearps, S., Kinner, S. A., Borschmann, R., Moran, P., Patton, G., 2015. Associations between psychotic symptoms and substance use in young offenders. Drug and Alcohol Review, n/a-n/a.

Ding, Y., Lin, H., Zhou, L., Yan, H., He, N., 2014. Adverse childhood experiences and interaction with methamphetamine use frequency in the risk of methamphetamineassociated psychosis. Drug Alcohol Depend. 142, 295-300.

Glasner-Edwards, S., Mooney, L. J., 2014. Methamphetamine psychosis: Epidemiology and management. CNS Drugs. 28, 1115-1126.

Gossop, M., Darke, S., Griffiths, P., Hando, J., Powis, B., Hall, W., Strang, J., 1995. The Severity of Dependence Scale (SDS): psychometric properties of the SDS in English and Australian samples of heroin, cocaine and amphetamine users. Addiction. 90, 607-614.

Grezellschak, S., Lincoln, T. M., Westermann, S., 2015. Cognitive emotion regulation in patients with schizophrenia: Evidence for effective reappraisal and distraction. Psychiatry Res.

Griffith, J. D. (1970). Experimental psychosis induced by the administration of damphetamine. . In Costa E. \& Garattini S. (Eds.), Amphetamine and Related Compounds (Vol. p. 876-904). New York: Raven Press. 
Hall, W., Hando, J., Darke, S., Ross, J., 1996. Psychological morbidity and route of administration among amphetamine users in Sydney, Australia. Addiction. 91, 81-87.

Hides, L., Dawe, S., McKetin, R., Kavanagh, D. J., Young, R. M., Teesson, M., Saunders, J. B., 2015. Primary and substance-induced psychotic disorders in methamphetamine users. Psychiatry Res. 226, 91-96.

Iwanami, A., Sugiyama, A., Kuroki, N., Toda, S., Kato, N., Nakatani, Y., Horita, N., Kaneko, T., 1994. Patients with methamphetamine psychosis admitted to a psychiatric hospital in Japan. A preliminary report. Acta Psychiatr Scand. 89, 428-432.

Lecomte, T., Mueser, K. T., MacEwan, W., Thornton, A. E., Buchanan, T., Bouchard, V., Goldner, E., Brink, J., Lang, D., Kang, S., Barr, A. M., Honer, W. G., 2013.

Predictors of persistent psychotic symptoms in persons with methamphetamine abuse receiving psychiatric treatment. J Nerv Ment Dis. 201, 1085-1089.

Lukoff, D., Nuechterlein, K. H., Ventura, J., 1986. Manual for the expanded Brief Psychiatric Rating Scale. Schizophr Bull. 12, 594-602.

McGrath, J. J., Saha, S., Al-Hamzawi, A., et al., 2015. Psychotic experiences in the general population: A cross-national analysis based on 31261 respondents from 18 countries. JAMA Psychiatry. 72, 697-705.

McKetin, R., Lubman, D. I., Baker, A. L., Dawe, S., Ali, R. L., 2013. Dose-related psychotic symptoms in chronic methamphetamine users: evidence from a prospective longitudinal study. JAMA Psychiatry. 70, 319-324.

McKetin, R., McLaren, J., Lubman, D. I., Hides, L., 2006. The prevalence of psychotic symptoms among methamphetamine users. Addiction. 101, 1473-1478.

McKetin, R., Najman, J., Baker, A., Lubman, D., Dawe, S., Ali, R., Lee, N., Mattick, R., Mamun, A., 2012. Evaluating the impact of community-based treatment options on methamphetamine use: findings from the Methamphetamine Treatment Evaluation Study (MATES). Addiction. 107, 1998-2008

Mueser, K. T., Crocker, A. G., Frisman, L. B., Drake, R. E., Covell, N. H., Essock, S. M., 2006. Conduct disorder and antisocial personality disorder in persons with severe psychiatric and substance use disorders. [see comment]. Schizophr Bull. 32, 626-636.

Niemi-Pynttari, J., Sund, R., Putkonen, H., Vorma, H., Wahlbeck, K., Pirkola, S., 2013. Substance-induced psychosis converting into schizophrenia: A register-base study of 18,478 Finnish inpatient cases. J Clin Psychiatry. 74, e94.

Nock, M. K., Kazdin, A. E., Hiripi, E., Kessler, R. C., 2006. Prevalence, Subtypes, and Correlates of DSM-IV Conduct Disorder in the National Comorbidity Survey Replication. Psychol Med. 36, 699-710.

Robins, L. N., Helzer, J. E., Ratcliff, K. S., Seyfried, W., 1982. Validity of the diagnostic interview schedule, version II: DSM-III diagnoses. Psychol Med. 12, 855-870.

Salo, R., Fassbender, C., Iosif, A.-M., Ursu, S., Leamon, M. H., Cameron, C., 2013. Predictors of methamphetamine psychosis: History of ADHD-relevant childhood behaviors and drug exposure. Psychiatry Res. 210, 10.1016/j.psychres.2013.1006.1030.

Sato, M., 1983. Long-lasting hypersensitivity to methamphetamine following amygdaloid kindling in cats: the relationship between limbic epilepsy and the psychotic state. Biol Psychiatry. 18, 525-536.

Schuckit, M. A., 2006. Comorbidity between substance use disorders and psychiatric conditions. Addiction. 101 Suppl 1, 76-88.

Stata Corporation. (2014). Stata/SE 12.1 for Windows. College Station, Texas: StataCorp LP.

Sulaiman, A. H., Said, M. A., Habil, M. H., Rashid, R., Siddiq, A., Guan, N. C., Midin, M., Nik Jaafar, N. R., Sidi, H., Das, S., 2014. The risk and associated factors of methamphetamine psychosis in methamphetamine-dependent patients in Malaysia. Compr Psychiatry. 55 Suppl 1, S89-94.

Tomiyama, G., 1990. Chronic schizophrenia-like states in methamphetamine psychosis. Jpn J Psychiatry Neurol. 44, 531-539. 
Tsuang, M. T., Simpson, J. C., Kronfol, Z., 1982. Subtypes of drug abuse with psychosis. Demographic characteristics, clinical features, and family history. Arch Gen Psychiatry. 39, 141-147.

van Os, J., 2014. The many continua of psychosis. JAMA Psychiatry. 71, 985-986.

Ventura, J., Green, M. F., Shaner, A., Liberman, R. P., 1993. Training and quality assurance with the Brief Psychiatric Rating Scale: 'the drift busters'. International Journal of Methods in Psychiatric Research. 3, 221-244.

Zavos, H. M., Freeman, D., Haworth, C. M., McGuire, P., Plomin, R., Cardno, A. G., Ronald, A., 2014. Consistent etiology of severe, frequent psychotic experiences and milder, less frequent manifestations: a twin study of specific psychotic experiences in adolescence. JAMA Psychiatry. 71, 1049-1057. 
Table 1. Characteristics of participants by vulnerability to psychotic symptoms

\begin{tabular}{|c|c|c|c|c|c|}
\hline & $\begin{array}{l}\text { No psychotic } \\
\text { symptoms } \\
(\mathrm{n}=110)\end{array}$ & $\begin{array}{l}\text { Transient } \\
\text { psychosis } \\
(\mathrm{n}=85)\end{array}$ & $\begin{array}{l}\text { Persistent } \\
\text { psychosis } \\
(\mathrm{n}=37)\end{array}$ & $\begin{array}{l}\mathrm{P} \text { value for } \\
\text { overall test }\end{array}$ & $\begin{array}{l}\text { Total sample } \\
(\mathrm{n}=232)\end{array}$ \\
\hline Male $(\%)$ & 7 & $82 *$ & 76 & .057 & 74 \\
\hline Age (median) & 31.5 & 31 & 31 & .361 & 31 \\
\hline Immigrant status (\%) & 7 & 15 & 8 & .168 & 10 \\
\hline $\begin{array}{l}\text { Family history of psychotic disorder }(\%) \\
\text { Comorbid disorders }(\%)\end{array}$ & 15 & 26 & $35^{*}$ & .029 & 22 \\
\hline Conduct disorder & 66 & $85^{* *}$ & $84^{*}$ & .006 & 76 \\
\hline Major Depression & 55 & 64 & $78^{*}$ & .043 & 63 \\
\hline Social Phobia & 15 & $38 * * *$ & $46 * * *$ & $<.001$ & 29 \\
\hline Panic Disorder & 18 & $39^{* *}$ & $62 * * * \S$ & $<.001$ & 34 \\
\hline Self-reported ADHD & 19 & 16 & 11 & .504 & 17 \\
\hline \multicolumn{6}{|l|}{ Methamphetamine use history } \\
\hline Age first use (median) & 17 & 16 & 16 & .096 & 31 \\
\hline Years of use (median) & 12 & 11 & 12 & .850 & 11.5 \\
\hline \multicolumn{6}{|l|}{ Methamphetamine use at baseline } \\
\hline SDS score (median) & 9 & 9 & $11^{* *}$ & .016 & 9 \\
\hline Days of use in past month (median) & 14 & 16 & 15 & .282 & 15 \\
\hline Injecting $(\%)$ & 74 & 69 & 73 & .800 & 74 \\
\hline \multicolumn{6}{|l|}{ Other drug use ${ }^{\mathrm{a}}(\%)$} \\
\hline Tobacco & 94 & 93 & 89 & .663 & 93 \\
\hline Alcohol & 74 & 66 & 70 & .502 & 70 \\
\hline Cannabis & 78 & 81 & 78 & .867 & 78 \\
\hline Ecstasy & 22 & 26 & 22 & .775 & 24 \\
\hline Cocaine & 19 & 26 & 32 & .215 & 27 \\
\hline Heroin & 22 & 22 & 16 & .725 & 22 \\
\hline Other opioids & 27 & 24 & 22 & .731 & 27 \\
\hline Inhalants & 8 & 7 & 8 & .995 & 7 \\
\hline Antidepressants & 24 & 20 & 22 & .830 & 22 \\
\hline Benzodiazepines & 35 & 45 & 35 & .319 & 41 \\
\hline
\end{tabular}

$* \mathrm{p}<0.05, * * \mathrm{p}<0.01, * * * \mathrm{p}<0.001$ relative to no psychotic symptoms; $\S \mathrm{p}<0.05$ relative to transient psychosis.

${ }^{a}$ Other drug use is any use of the drug in the past month at the baseline interview. 